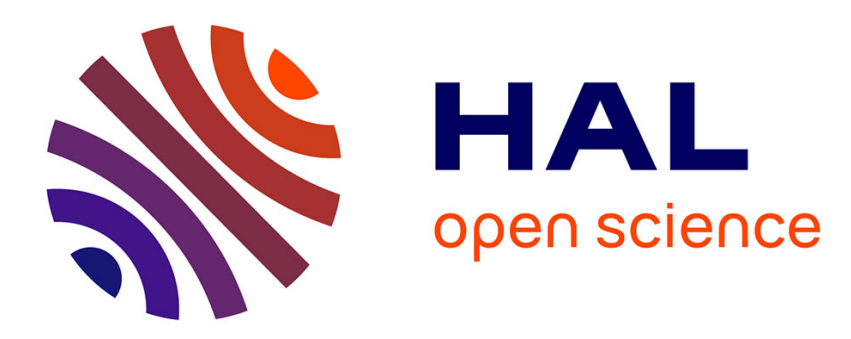

\title{
Discovering Connected Objects in the Criminal Investigations
}

François Bouchaud, Thomas Vantroys, Gilles Grimaud, Pierrick Buret

\section{To cite this version:}

François Bouchaud, Thomas Vantroys, Gilles Grimaud, Pierrick Buret. Discovering Connected Objects in the Criminal Investigations. COINS 2020, Aug 2020, Barcelona, Spain. hal-02934143

\section{HAL Id: hal-02934143 \\ https://hal.science/hal-02934143}

Submitted on 9 Sep 2020

HAL is a multi-disciplinary open access archive for the deposit and dissemination of scientific research documents, whether they are published or not. The documents may come from teaching and research institutions in France or abroad, or from public or private research centers.
L'archive ouverte pluridisciplinaire HAL, est destinée au dépôt et à la diffusion de documents scientifiques de niveau recherche, publiés ou non, émanant des établissements d'enseignement et de recherche français ou étrangers, des laboratoires publics ou privés. 


\title{
Discovering Connected Objects in the Criminal Investigations
}

\author{
François Bouchaud*, Thomas Vantroys ${ }^{\dagger}$, Gilles Grimaud ${ }^{\dagger}$, Pierrick Buret ${ }^{\ddagger}$ \\ *IRCGN - Forensic science laboratory, Gendarmerie Nationale \\ francois.bouchaud@gendarmerie.interieur.gouv.fr \\ ${ }^{\dagger}$ Univ. Lille, CNRS, Centrale Lille, UMR 9189 - CRIStAL F-59000 Lille, France \\ ${ }^{\ddagger} \mathrm{C} 3 \mathrm{~N}$ - National cyber-crime unit, Gendarmerie Nationale
}

\begin{abstract}
More and more things around us are becoming digital and connected to the Internet. This market ranges from smart devices, wellness and health control to smart cities. This development offers to malicious parties the possibility of carrying out attacks, directly impacting the consumers of these new services. Thus, the connected objects are actors or witnesses of events that have occurred. This opens up a challenge for security and forensic investigations on the Internet of Things (IoT).

In this article, we present the problem of finding connected objects on an offence scene. In the absence of a technical solution, the investigators limit themselves to a manual search. Hidden objects are often neither detected nor found. Thus, we aim to give a clear and precise image of the current devices. We also want to determine their position.

This work focuses on the study of the digital signature of the scene and the radio frequency characteristics of the objects. To understand the electromagnetic environment, we use a software defined radio (SDR) and we develop several tools: a sensor for a single protocol and a mesh network of sensors. The SDR returns the used frequencies. The single receiver offers a global mapping of the environment on a given protocol. The multi-sensor mesh network gives a precise and targeted vision of the infrastructure connected to several protocols and frequencies. We propose to assess the relevance of the measurement methods in relation to operational needs, on the basis of a use case and feedbacks.

Index Terms-Internet of Things, IoT Investigations, Detection and Localization.
\end{abstract}

\section{INTRODUCTION}

The rapid growth of the Internet of Things (IoT) market offers the digitalization of the life. Every person creates a digital footprint saved by devices. This collected information is valued through the infrastructure to offer new services. In legal proceedings, this data can be used in a court to date an event, to confuse a murderer or a testimony. Several real cases incorporating connected objects have recently made the headlines. For instance, the log from a Global Positioning System (GPS) watch was used to resolve a murder case [1]. In an Arkansas murder case, the Amazon Echo device acted as a witness [2]. In an other case, the correspondence between the victim's FitBit data and information from the home automation system highlighted the murder of a woman [3]. On the operational side, connected objects must be taken into account during a police intervention.

Forensic investigators are well trained to identify and analyse an electronic medium, such as a mobile phone or a computer. However, IoT devices come in many forms. They are not always visible. Thus, the research process becomes more complex. Moreover, they do not broadcast continuously, to limit energy consumption. Investigators may miss the presence of communication systems. This article deals with the problem of discovering connected objects and their position. It consists in studying the behaviour of an environment by analysing radio frequency communications. We provide several forensic tools. This work is experimentally tested to analyse its robustness, in an operational context in France.

Section II highlights the needs to detect and locate a connected object as part of a forensic investigation; Section III covers previous work in the field of radio frequency location; Section IV describes the operational tools; Section V tests them in a laboratory use cases, in particular to determinate their effectiveness; Section VI comments them in real cases; Section VII provides the conclusion of the paper and the further work of this research.

\section{BACKGROUND}

This section defines the detection and localization needs of a connected object. Besides, it presents the encountered difficulties.

\section{A. Forensic Need}

The criminal event is singular, unusual, and irreversible in time and space. The observed trace stands out from the current activity in all objectivity and neutrality. The goal is to describe the case in its uniqueness. The materiality of the trace makes it possible to measure and determine its own characteristics. It compares to other known data. This primary element witnessing a past fact is put into perspective with its peers and the environment. This information becomes clues in the judicial process. It establishes the circumstances and the criminal facts. Once they are structured and appropriated by the judge, they become expert evidence for the criminal trial. The criminal investigation is based on the abduction principle developed in [4]. It is a question of establishing the most probable causes of the observed event. Hypotheses are then asserted on the crime by studying the link with a probable cause. For example, relationships between tools traces, the operating mode and the knowledge of the materials characteristics [5] allow building reasoning on a given burglary. Bayes' theorem on the probability of causes takes into account bias and uncertainty [6].

The IoT infrastructure is made up locally of heterogeneous objets. The investigator searches for available traces that are eventually linked to these physical devices. In the absence of visual identification, the study of the digital footprint of the scene reveals devices. These devices can be small, low power and energy self-sufficient. Similarly, they can issue a weak digital signature, through intermittent and time-limited communications. Limited resources cap the amount and type of data that will be transmitted. These elements constitute a constraint in the search for connected objects.

\section{B. Legal Grounds and Privacy}

Without legal grounds, the capture of personal data is considered as a violation of privacy in many countries. Investigators may face this problem when investigating a scene of an offence. They cannot be satisfied with detecting only the radio frequency signatures of objects. Thus, information from objects located outside the study area must be discriminated. It is therefore necessary to locate the target equipment in order to be able to link it to the offending environment.

Faced with these different needs and findings, it is necessary to think about technical solutions to find connected objects in a study area. 


\section{Previous Works}

In this section, we propose a state of the art of work carried out on the detection and the localization of connected objects and the limits of these approaches in a crime scene intervention.

There are existing works on indoor and outdoor positioning. The majority of methods are based on the characteristics of radiofrequency transmissions like the Received Signal Strength Indicator (RSSI), the Time Difference of Arrival (TDOA), the Angle of Arrival (AoA), the phase shift, etc.This section focuses on RSSI and phase shift. The other solutions (TDOA, AoA, hop count, etc.) are more complicated to implement in an operational environment, highly constrained. For example, TDOA requires network synchronization.

Radio receiver can provide an estimate of the power received. The strength of a device radio signal decreases as the distance from the source increases. This phenomenon is explained by the interactions between the wave and the propagation medium. There are several models to describe this relationship: the Friis' equation [7], the Two Ray Ground Reflection model and the Shadowing model [8]. The path loss exponent is linked to the characteristics of the environment in which it goes through [9].

Several physical factors must also be taken into account, such as temperature measurements. The presence of several floors affects the measurements. The relative position of the antennas conditions the detected power level. Thus, a profiling step is carried out upstream on the study environment. According to the detected protocols, the measurements are carried out over long periods. This is particularly the case for protocols transmitting over short discontinuous periods.

On the basis of these information, it is possible to deduce the distance travelled by the signal. The literature contains a multitude of solutions for estimating the position of a communicating node from RSSI [10] [11] [12]. Several articles are interested in locating objects connected via RSSI for Wi-Fi [13], Bluetooth [14] and ZigBee [15] protocols.

So, we propose several solutions for searching for connected objects, according to their emission characteristics. Each responds to specific operational needs.

\section{RAdio-Frequency Forensic RESEARCH TOOLS}

In this section, we present three forensic tools we are developing to find connected objects. Their uses are defined according to operational constraints or the implementation strategy.

\section{A. Software Defined Radio}

The Software Defined Radio (SDR) [16] is used to determine the emissions present in the study area. It scans the entire frequency spectrum and determines used one. Depending on the result, we can quickly and passively know if there are active objects. So, this solution offers the possibility of carrying out doubt inquiries.

We have not developed a software brick dedicated to localization. This choice is motivated by questions of cost of reprogramming and deployment of a SDR solution. Localization is carried out by two dedicated tools: a single receiver or a multi-sensor mesh network. Depending on the frequency and protocols found with the SDR, we will choose the most suitable tool. It also offers more precise measurements of the environment.

\section{B. Single Receiver}

The single receiver is a variant of the SDR approach based on a chip dedicated to a single protocol. A mobile sensor scans the study area over a known frequency range. It gives a global picture of active devices using the same protocols. Measurements are passive without interference or injection of payload, in order to respect the legal aspects. They are carried out by moving into the target area. Localization is mainly done by studying the value of RSSI.

The receiver is made up of four parts: sector antennas at a fixed frequency, an auto-location system composed of Global Positioning System (GPS), accelerometers and gyroscopes, an operating system, and a battery.

To improve the efficiency and reduce the target area, the measurements are coupled with information from networks such as Global System for Mobile Communications (GSM) and Wi-Fi, in particular in the search for a mobile phone. It gives a vision of the interactions and dependencies between connected objects with identical characteristics. The use of drones containing the single receiver provides a measure of topologically hilly spaces.

\section{Multi-Sensor Mesh Network}

The multi-sensor mesh network is based on the static study of a specific area from several synchronized sensors. They have the particularity of belonging to the same mesh network. They are controlled by a central node. This solution allows precise positioning of connected objects in three dimensions by relying on RSSI and the phase shift between the sensors.

The multi-sensor is made up of six parts: sector antennas at fixed frequency, an auto-location system composed of GPS, accelerometers and gyroscopes, an operating system, a battery, a mesh communication system, and a synchronization module. It is accompanied by a system dedicated to calibrating the sensor. It assesses external information such as temperature, pressure, aperture and movement. The antenna module is composed of several antennas observing 120 degrees (horizontal) by 15 degrees (vertical) sectors. In our study, we investigate omnidirectional antennas polarized vertically with respect to the ground or quarter-wave antennas. A level comparator gives the attenuation of each of them relatively to the signal of higher amplitude, after discrimination by the identifier of the Wi-Fi channel. They have the advantage of being directional and of small dimensions. A magnetometer is used for auto-location in addition to $3 \mathrm{D}$ visualization. It allows to enter the site configuration and the $3 \mathrm{D}$ position of each sensor.

The sensors are located at least at three ends of the study area. To improve the accuracy, a sensor is located at the centre. A signal is transmitted to determine the positioning of the sensors and their calibrations. The synchronization of the sensor clock is regulated by radio frequency. This network infrastructure analyses the entire frequency spectrum of the Industrial, Scientific and Medical (ISM) radio bands. The gathered information guide the deployment of new sensors configured on target protocols. These newcomers improve the accuracy of locating discovered objects. They also make it easier to find hidden objects. The proposed approach is to perform a global measurement of the frequency activity. Then, we carry out concentric studies in a closed environment. The speed and the quality of synchronization between the sensors as well as the continuity of communications have a significant impact on the achieved measurement. The measurements are also coupled to network information such as GSM and Wi-Fi. They give an instant measurement on all protocols, standardized or not. For example, this equipment can be used in the search for objects emitting on prohibited frequencies.

\section{Evaluation Strategies}

The time measurement criteria and the knowledge of the used protocols are the determining elements to favour the choice of the tool. Indeed, time can be constrained by operational or legal reasons. 


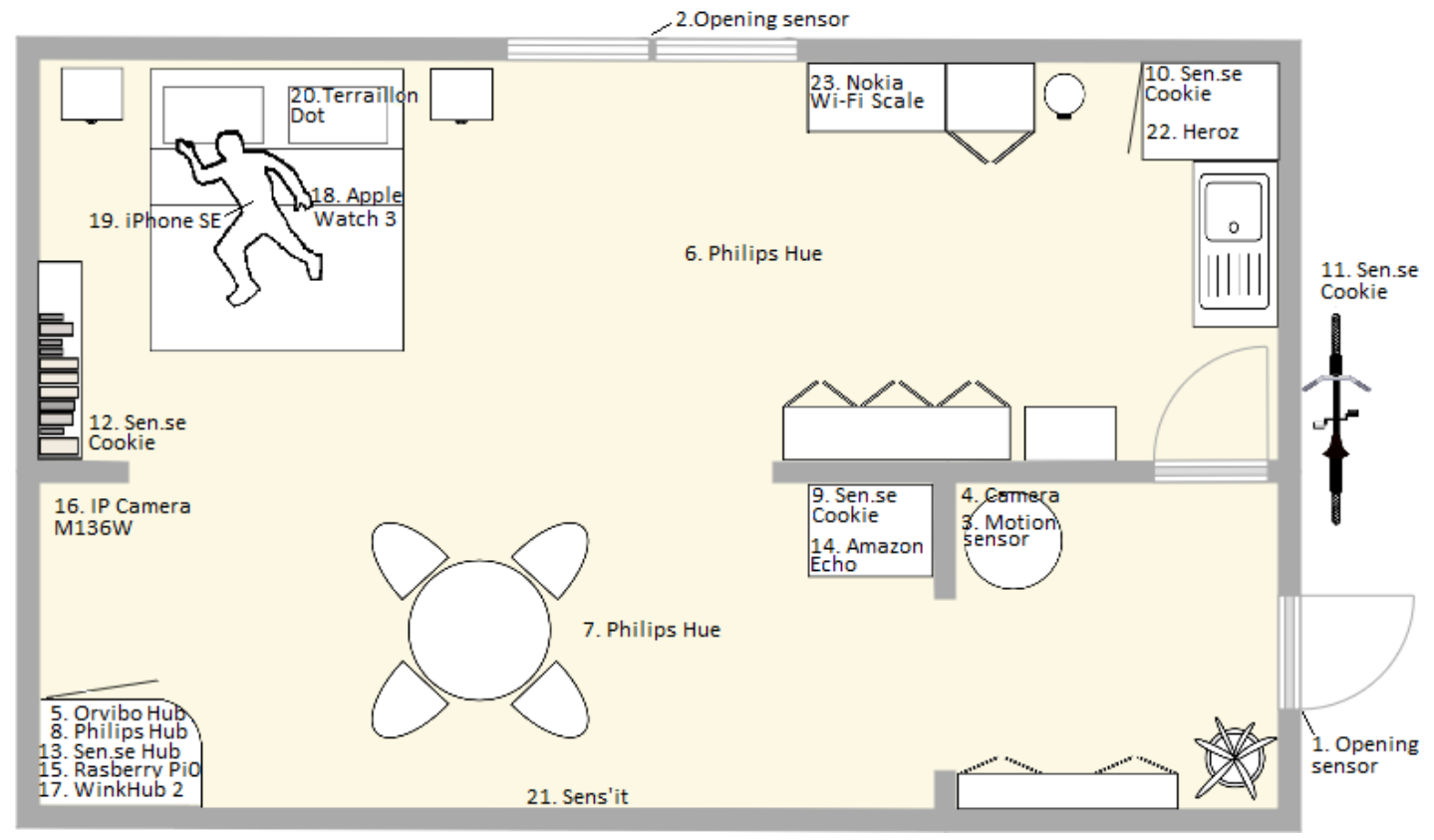

Fig. 1. Home layout with the IoT devices

We can cite the cases of a search during police custody. Conversely, forensic investigators have more time to deal with a crime scene. Furthermore, the characteristics of the field can also guide the choice of one method.

The single receiver is often used when the connected object or the communication protocol is known, such as Wi-Fi or Bluetooth. This solution is very effective in the treatment of large study areas for a limited time. The multi-sensor mesh network is more complex to deploy, due to the synchronization problem. However, it gives a precise and targeted vision of the infrastructure connected to several protocols and frequencies, known or not. In the management of a crime scene, this method is preferred. The two methods can be combined to optimize the study of the target, according to an approach that goes from general towards a particular point of view.

So, we propose to assess the relevance of the measurement methods in relation to operational needs on the basis of a use case and feedback.

\section{EVAlUATION OF ForEnSIC TOOLS ON AN INVESTIGATIVE EXERCISE}

This section assesses the forensic tools on an investigation exercise. We seek to appreciate the advantage brought by these tools in the search of connected objects. Two parameters are evaluated: the number of found objects and the discovery time. This laboratory exercise is inspired by real cases encountered by forensic investigators.

\section{A. Presentation of the Scenario and the Environment}

The scenario of this specific exercise is relative to the discovery of a corpse in an apartment. The apartment covers $45 \mathrm{~m}^{2}(5 \mathrm{~m} \mathrm{x} 9 \mathrm{~m})$. It includes three separate rooms: an entrance (room 1), a bedroom (room 2) and a living room (room 3) (Fig. 1).

This flat contains many connected objects belonging to several IoT architectures. It has a home automation system from an Orvibo kit. It contains two opening sensors (1 and 2); and a motion sensor (3) coupled to a Wi-Fi camera (4). This kit is located in the room 1 and on two exterior openings. This infrastructure communicates via
ZigBee to a dedicated hub (5), located in the room 3. The home automation system is also made of Philips brand connected bulbs (6 and 7) with its dedicated hub (8). They are located in rooms 2 and 3 of the apartment. Otherwise, four Sen.se Cookies are hidden in the different rooms. They transform household objects into connected objects. In our case, Cookies follow the water supply level of the coffee machine (9), the ambient temperature (10), the position of the bicycle (11) and the physical activity of the victim (12). All these objects are connected with a proprietary protocol to Mother Sen.se (13), in room 3. Thus, these different hubs, an Amazon Echo (14), a RaspberryPi0 (15) and an IP camera M136W (16) are connected to the Internet by WinkHub 2 (17).

The victim is lying on the bed in room 2. She has an Apple Watch series 3 (18) on her right arm and an iPhone SE (19) in her pocket. Hidden in the bed, there is a sleep sensor named Terraillon Dot (20). The apartment contains other objects such as Sens'it (21), a Heroz bracelet (22) and a Nokia Wi-Fi scale (23). Fig. 2 represents the communication architecture.

\section{B. Description of the conditions and parameters of the experiment}

Experimentation makes it possible to study the performance and effectiveness of the proposed solutions. We define two measurement criteria. The first is a ratio between the number of objects detected compared to those present. The second calculates the time to discover objects. The scenario includes heterogeneous connected objects: home automation, health, virtual assistants, etc. These objects communicate via different protocols (Wi-Fi, Bluetooth, ZigBee, etc.).

We perform a first series of measurements with SDR equipment to determine the used frequencies. In order not to alter the study area, the measurements are made only around the apartment, as in a real case of investigation. Thus in this experiment, we are not able to discriminate the objects present outside the scene. We carry out a second series of measurements with the single receiver. It is made at different heights to integrate the third dimension. Finally, we realize a third series with the multi-sensor mesh network. We position three measurement sensors around the experimental scene. A fourth sensor 


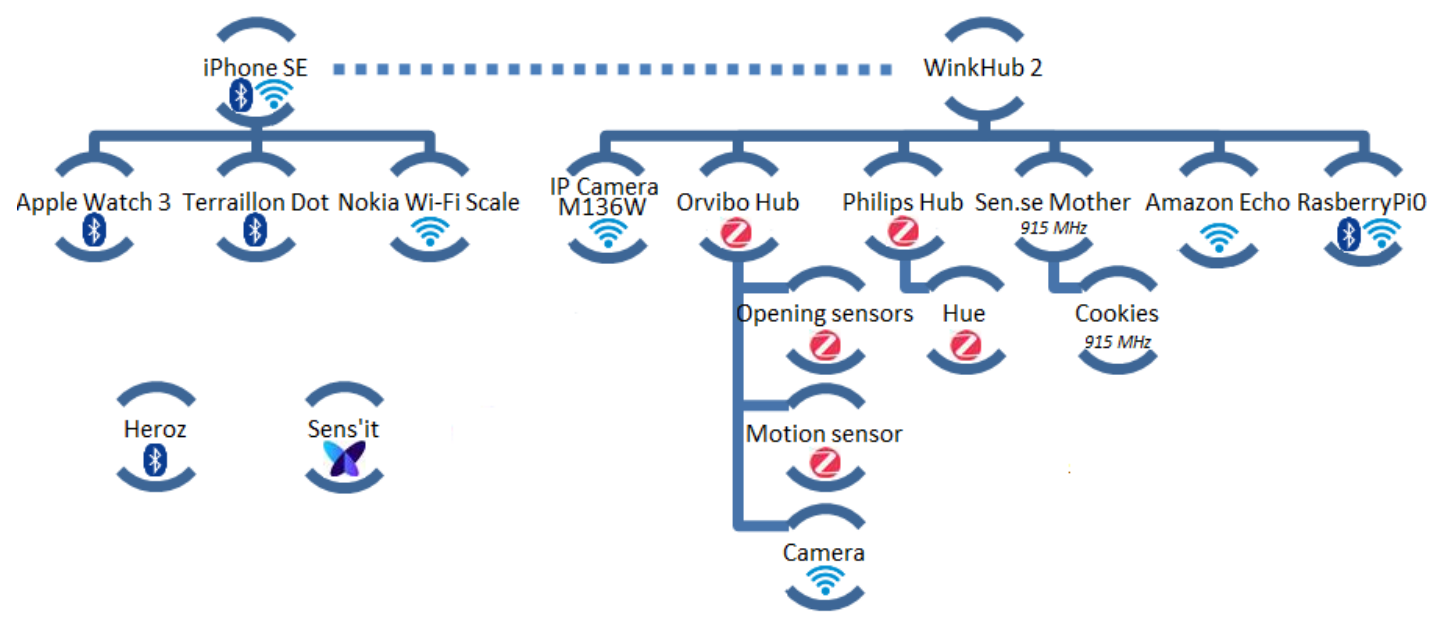

Fig. 2. Global architecture of the IoT environment

TABLE I

Tools Performance AND EFFiciency Study

\begin{tabular}{|c|c|c|c|c|c|c|c|}
\hline & Device & Manufacturer & No equipment & SDR & Single Receiver & Multi-Sensor Mesh Network & All solutions \\
\hline 1 & Opening Sensor & Orvibo & No & No & No & Detect/ Locate & Detect/ Locate \\
\hline 2 & Opening Sensor & Orvibo & No & No & No & No & No \\
\hline 3 & Motion Sensor & Orvibo & No & No & No & Detect/ Locate & Detect/ Locate \\
\hline 4 & Camera & Orvibo & No & Detect & Detect/ Locate & Detect/ Locate & Detect/ Locate \\
\hline 5 & Hub & Orvibo & Locate & No & No & Detect/ Locate & Detect/ Locate \\
\hline 6 & Electric bulb & Philips Hue & No & No & No & No & No \\
\hline 7 & Electric bulb & Philips Hue & No & No & No & No & No \\
\hline 8 & Hub & Philips Hue & Locate & Detect & Detect/ Locate & Detect/ Locate & Detect/ Locate \\
\hline 9 & Cookie & Sen.se & No & Detect & No & Detect/ Locate & Detect/ Locate \\
\hline 10 & Cookie & Sen.se & No & Detect & No & Detect/ Locate & Detect/ Locate \\
\hline 11 & Cookie & Sen.se & No & Detect & No & Detect/ Locate & Detect/ Locate \\
\hline 12 & Cookie & Sen.se & No & Detect & No & Detect/ Locate & Detect/ Locate \\
\hline 13 & Mother & Sen.se & No & Detect & No & Detect/ Locate & Detect/ Locate \\
\hline 14 & Echo Spot & Amazon & Locate & Detect & Detect/ Locate & Detect/ Locate & Detect/ Locate \\
\hline 15 & $\mathrm{PiO}$ & Rasberry & Locate & Detect & Detect/ Locate & Detect/ Locate & Detect/ Locate \\
\hline 16 & IP Camera M136W & & No & Detect & Detect/ Locate & Detect/ Locate & Detect/ Locate \\
\hline 17 & WinkHub 2 & Wink & Locate & Detect & Detect/ Locate & Detect/ Locate & Detect/ Locate \\
\hline 18 & Watch 3 & Apple & Locate & Detect & Detect/ Locate & Detect/ Locate & Detect/ Locate \\
\hline 19 & iPhone SE & Apple & Locate & Detect & Detect/ Locate & Detect/ Locate & Detect/ Locate \\
\hline 20 & Dot & Terraillon & No & No & No & No & No \\
\hline 21 & Sens'it 2.1 & Sens'it & No & No & No & Detect/ Locate & Detect/ Locate \\
\hline 22 & Bracelet & Heroz & No & No & No & No & No \\
\hline 23 & Wi-Fi Scale & Nokia & No & Detect & Detect/ Locate & Detect/ Locate & Detect/ Locate \\
\hline \multicolumn{3}{|c|}{ Detect } & $0 \%$ & $60 \%$ & $40 \%$ & $78 \%$ & $78 \%$ \\
\hline \multicolumn{3}{|c|}{ Locate } & $30 \%$ & $0 \%$ & $40 \%$ & $78 \%$ & $78 \%$ \\
\hline \multicolumn{3}{|c|}{ Average localization accuracy (meter) } & 0 & 0 & 2.2 & 1.1 & 1.1 \\
\hline \multicolumn{3}{|c|}{ Average duration (min): } & 0 & 1 & 2 & 33 & 36 \\
\hline \multicolumn{3}{|c|}{ - acquisition phase } & 45 & 18 & 24 & 54 & 44 \\
\hline \multicolumn{3}{|c|}{ - uninstall phase } & 0 & 1 & 2 & 33 & 36 \\
\hline \multicolumn{3}{|c|}{ Total duration (min) } & 45 & 20 & 28 & 120 & 116 \\
\hline
\end{tabular}

is placed at the centre of the apartment. For the last two series, we exclude the communication coming frome the outside. This exclusion is based on the measurement of the location of the emitting sources. The analysis is repeated several times over several days with identical climatic conditions.

We are also doing a manual search for devices in this flat. Moreover, these experiments are carried out by teams who do not know the nature and the number of present objects.

\section{Results and Discussion}

Table I contains the different objets of the crime scene. For each object, we indicate if it is detected by manual inspection or by a tool. The performance ratio is equal to the ratio between the number of found/detected objects and the whole number of objects. The efficiency rate is estimated on the basis of the duration of acquisition of the objects. We are studying the location accuracy of the measurements.

In order to install the fourth sensor of the multi-sensor mesh network, one must enter into the apartment. This action will generate ZigBee communications between the opening sensor and the Orvibo hub. This event will be recorded and detected by the network. With a single receiver, we do not perceive this signature.

We observe that the tools provides an opportunity to find more 
TABLE II

CHARACTERISTICS OF THE ENVIRONMENT FOR THE USE OF TOOLS

\begin{tabular}{|c|c|c|c|c|c|c|c|c|}
\hline Characteristic/ Method & $\begin{array}{l}\text { Number } \\
\text { of } \\
\text { missions }\end{array}$ & $\begin{array}{l}\text { Urban } \\
\text { (buildings) }\end{array}$ & $\begin{array}{l}\text { Suburban (city } \\
\text { with house and } \\
\text { small buildings) }\end{array}$ & Countryside & Village & Forest & Mountain & $\begin{array}{l}\text { Sensitive } \\
\text { area }\end{array}$ \\
\hline No equipment & 12 & $33.33 \%$ & $8.33 \%$ & $16.67 \%$ & $41.67 \%$ & $0.00 \%$ & $0.00 \%$ & $0.00 \%$ \\
\hline SDR & 34 & $14.71 \%$ & $5.88 \%$ & $32.35 \%$ & $11.76 \%$ & $35.29 \%$ & $0.00 \%$ & $0.00 \%$ \\
\hline Single Receiver & 200 & $37.00 \%$ & $29.50 \%$ & $11.00 \%$ & $22.00 \%$ & $0.50 \%$ & $0.00 \%$ & $0.00 \%$ \\
\hline Multi-Sensor Mesh Network & 110 & $15.55 \%$ & $20.00 \%$ & $24.55 \%$ & $16.36 \%$ & $18.18 \%$ & $0.00 \%$ & $6.36 \%$ \\
\hline All solutions & 44 & $11.36 \%$ & $9.09 \%$ & $6.82 \%$ & $6.82 \%$ & $11.36 \%$ & $18.18 \%$ & $36.36 \%$ \\
\hline
\end{tabular}

connected objects than a manual approach. The Terraillon Dot is not detected because Bluetooth communication is only active during manual data synchronization. No communication is detected between the lamps in the Philips Hue kit and its hub. Indeed, this requires the realization of an event from the control application. Sens'it based on the Sigfox protocol provides punctual and limited feedback. Thus, detection requires a long measurement over time. The Heroz bracelet is not connected to a network. We cannot detect it.

These tools are tested by investigator field units on forensic missions. These results allow us to comfort the correctness of our laboratory results. They integrate the constraints linked to the environment.

\section{Evaluation in Real Cases}

To carry out this statistical study, we examine the last 400 relevant forensic missions to find connected objects by the French gendarmerie cyber-crime investigation unit. This analysis is carried out between November 2015 and November 2019.

\section{A. Analysis of the characteristics of the mission environment}

Table II shows the use of the tools developed according to the characteristics of the measurement area.

It should be read horizontally. Missions without equipment take place mainly in residential areas for urban and village environments. It represents three quarters of the studied cases. Indeed, the so-called manual method is limited in terms of efficiency for a large coverage area with hidden objects. SDR equipment meets this need for broad coverage of the countryside or the forest. It offers rapid resolution of doubts, especially in the context of an on-site intervention. The single sensor is mainly used in urban and suburban areas. It offers a general framing with the use of drones. It also provides a targeted search response when searching for known Wi-Fi and Bluetooth connected objects. The multi-sensor is used in heterogeneous areas. This result is explained by the ability of the tool to cover more different protocols and frequencies. However, its use is more restrictive. Mixed solutions are used in a complex environment. They respond to the problems of targeting and covering a large area.

Table III focuses on the geographic and climatic characteristics associated to the terrain.

Several characteristics are highlighted from the analysis of the results. The coverage of a residential area and its surroundings is estimated to approximately $2.5 \mathrm{~km}^{2}$. Spaces without accommodation are on average 6 to $10 \mathrm{~km}^{2}$. The mountain due to the characteristics of the relief limits the propagation of the waves and the measurement with our tools. The so-called sensitive environments are made up of large areas protected from urbanization and civil activity.

B. Analysis of the use of equipment on the discovery of connected objects on a mission

1) Number of radio signals detected per mission (not modulated or partially): Table IV gives an overview of the number of signals
TABLE III

CHARACTERISTICS OF THE ENVIRONMENT

\begin{tabular}{|l|l|l|l|l|}
\hline & $\begin{array}{l}\text { Average } \\
\text { covered } \\
\text { area }\left(\mathrm{km}^{2}\right)\end{array}$ & $\begin{array}{l}\text { Minimum } \\
\text { Tempera- } \\
\text { ture }\left(\mathrm{T}^{\circ} \mathrm{C}\right)\end{array}$ & $\begin{array}{l}\text { Maximum } \\
\text { Tempera- } \\
\text { ture }\left(\mathrm{T}^{\circ} \mathrm{C}\right)\end{array}$ & Rain/Snow \\
\hline Urban & 2.61 & -4 & 38.1 & $16 \%$ \\
Suburban & 2.34 & -3 & 39.5 & $22 \%$ \\
Countryside & 6.5 & -7 & 38.6 & $25 \%$ \\
Village & 2.8 & -2 & 36.7 & $20 \%$ \\
Forest & 10.7 & -2.9 & 35.9 & $22 \%$ \\
Mountain & 3.7 & -7 & 35.2 & $28 \%$ \\
Sensitive area & 15 & -3 & 38 & $23 \%$ \\
\hline
\end{tabular}

detected per mission. This table confirms the presence of connected devices on each studied scene.

TABLE IV

Number of Signals DeteCted on A Mission By EQUipMENT

\begin{tabular}{|l|l|l|l|}
\hline & Minimum & Maximum & Average \\
\hline No equipment & 0 & 0 & 0 \\
SDR & 2 & 45 & 24 \\
Single Receiver & 15 & 2428 & 834 \\
Multi-Sensor Mesh Network & 9 & 2498 & 1237 \\
All solutions & 8 & 2615 & 1280 \\
\hline
\end{tabular}

2) Number of connected objects discovered per mission: Table $\mathrm{V}$ examines the number of connected objects during the different missions.

TABLE V

Number of OBJECTS FOUND ON A Mission By EQUIPMENT

\begin{tabular}{|l|l|l|l|}
\hline & Minimum & Maximum & Average \\
\hline No equipment & 0 & 22 & 6.04 \\
SDR & 0 & 22 & 5.9 \\
Single Receiver & 0 & 45 & 9.6 \\
Multi-Sensor Mesh Network & 0 & 60 & 9.2 \\
All solutions & 0 & 80 & 14.6 \\
\hline
\end{tabular}

3) Percentage of discoveries with equipment available for a mission: Table VI shows the performance of the used tools. Percentages refer to the number of objects found with the tools in addition to a manual approach. They allow us to assess the added value of field tools. In these different measurements, we are not able to know the real number of objects present. Thus, the performance ratio between the number of objects found and the real number is not calculated.

\section{Discussion}

The number of connected objects encountered during a mission can exceed 80 per target. In the absence of tools, the volume of objects found is divided by 2 . The search for connected objects is a challenge for investigators. It cannot be fully automated. In addition, it focuses on active objects with clearly emitted signals. Analyses of 
TABLE VI

PROPORTION OF ITEMS FoUnd ON A MISSION BY THE EQUIPMENT

\begin{tabular}{|l|l|l|l|l|}
\hline & $\begin{array}{l}\text { Manuel } \\
\text { discovery }\end{array}$ & $\begin{array}{l}\text { SDR } \\
\text { Single } \\
\text { Receiver }\end{array}$ & $\begin{array}{l}\text { Multi- } \\
\text { Sensor } \\
\text { Mesh } \\
\text { Network }\end{array}$ \\
\hline No equipment & $100 \%$ & - & - & - \\
SDR & $85 \%$ & $15 \%$ & - & - \\
Single Receiver & $64 \%$ & - & $36 \%$ & - \\
Multi-Sensor Mesh Network & $58 \%$ & - & - & $42 \%$ \\
All solutions & $47 \%$ & $5 \%$ & $28 \%$ & $20 \%$ \\
\hline
\end{tabular}

the communication protocols and of the infrastructure are necessary. The analyses can be coupled to a classification of devices. An effort of clarity must be done for the investigation. The results must be intelligible by non-specialized investigators.

The question of measurement time is a primordial and incompressible factor in the choice of an acquisition method. This criterion is difficult to quantify and to generalize for all cases encountered by the investigators. It breaks down into three stages: a deployment phase of the measurement tool, an acquisition phase followed by data processing and a device uninstall phase. The installation time for the single receiver is two minutes on average. For a multi-sensor mesh network, it takes about thirty minutes to an hour. This time factor will depend on the characteristics of the terrain and the area to be covered. Moreover, it can evolve depending on the addition or removal of measuring devices. The hardware removal time is the same as the deployment time. The data acquisition time in a single receiver is between ten minutes and twelve hours. On average, it is about an hour. For the multi-sensor mesh network, it oscillates between an hour and a week of measurement. The average is twentyfour hours. Processing is almost instantaneous for a single receiver. With the multi-sensor mesh network, it is ten minutes. This time takes into account the collection of the information, the processing and the display on a web portal. However, this publication of the results does not take priority over their collections.

The accuracy of the measurements is also an important factor in making easier to find objects in a complex environment. It depends on the used software and hardware. For example, the antennas of single receivers are omnidirectional. Those of multi-sensor mesh networks are directional. This measurement is also strongly disturbed by the experimental measurement conditions: the weather and the environmental characteristics. These constraints are integrated into the calculations of our solution. Thus, the single receiver has an accuracy of about 10 metres. The accuracy of the multi-sensor mesh network is about 1 metre. Measurements are more precise under optimal measurement conditions. They are defined by the following characteristics: indoors, with stable weather and knowledge of the distribution of the used protocols. Thus, we obtain very good results in Wi-Fi thanks to a good control of the propagation of the waves. The single receiver can increase the accuracy of around 1 metre and the multi-sensor mesh network around $80 \mathrm{~cm}$. Conversely for the ZigBee, the precision of the multi-sensor mesh network is 1 to 2 metres.

\section{CONCLUSION}

Introducing the Internet of Things (IoT) into the society provides more opportunities for forensic investigations. Connected objects are the actors and direct witnesses of events. However, the objects are heterogeneous, not always visible and identifiable. Faced to these findings, investigators need to know the presence of objects and their positions in a crime scene. In this article, we present several tools to search for these objects, based on their radio signatures. From the RSSI and the phase shift, the objects are located precisely. This article proposes to assess the use of these tools based on a laboratory exercise, and then on the feedbacks from 400 police missions.

The next stage of this research is to select and collect them. The challenge is to understand the infrastructure of connected objects to optimize the collection method without altering the stored data. This solution must meet the requirements of the field.

\section{REFERENCES}

[1] T. J., "How police unmasked "iceman" assassin behind one of britain's most notorious gangland murders," Liverpool Echo, no. 1, Jan 2019. [Online]. Available: https://www.liverpoolecho.co.uk/news/ liverpool-news/how-police-unmasked-iceman-assassin- 15649613

[2] C. N., "Arkansas judge drops murder charge in amazon echo case," CNN, no. 2, Dec 2017. [Online]. Available: https://edition.cnn.com/2017/11/ 30/us/amazon-echo-arkansas-murder-case-dismissed/index.html

[3] C. M., "Fitbit offers key clue to slain san jose woman's alleged 90-year-old killer," San Francisco Chronicle, no. 3, Sep. 2018. [Online]. Available: https://www.sfchronicle.com/crime/article/ Fitbit-offers-key-clue-to-slain-San-Jose-13266777.php

[4] C. S. Peirce, Collected Papers: Science and philosophy and Reviews, correspondence, and bibliography. Belknap Press of Harvard University Press, 1958, vol. 7, no. 4.

[5] K. Inman and N. Rudin, Principles and practice of criminalistics: the profession of forensic science. CRC Press, 2000, no. 6.

[6] B. Robertson and T. Vignaux, "Bayes' theorem in the court of appeal," The Criminal Lawyer, vol. 69, no. 7, pp. 4-5, 1997.

[7] M. Cypriani, "Géopositionnement wi-fi autocalibré en milieu hétérogène," Université de Franche-Comté, no. 10, 2012.

[8] T. S. Rappaport et al., Wireless communications: principles and practice. prentice hall PTR New Jersey, 1996, vol. 2, no. 11.

[9] S. C. Lam and K. Sandrasegaran, "Analytical and simulation performance of a typical user in random cellular network," arXiv preprint arXiv:1607.03280, no. 26, 2016.

[10] V. Daiya, J. Ebenezer, S. S. Murty, and B. Raj, "Experimental analysis of rssi for distance and position estimation," in 2011 International Conference on Recent trends in information technology (ICRTIT), no. 12. IEEE, 2011, pp. 1093-1098.

[11] E. Cassano, F. Florio, F. De Rango, and S. Marano, "A performance comparison between roc-rssi and trilateration localization techniques for wpan sensor networks in a real outdoor testbed." in 2009 Wireless Telecommunications Symposium, no. 13. IEEE, 2009, pp. 1-8.

[12] F. Dong, Y. Chen, J. Liu, Q. Ning, and S. Piao, "A calibrationfree localization solution for handling signal strength variance," in International Workshop on Mobile Entity Localization and Tracking in GPS-less Environments, no. 17. Springer, 2009, pp. 79-90.

[13] F. Lassabe, P. Canalda, and P. Chatonnay, "Geolocalisation wifi et modeles de prediction de la mobilite dans les reseaux multimedia," 2009.

[14] M. Altini, D. Brunelli, E. Farella, and L. Benini, "Bluetooth indoor localization with multiple neural networks," in IEEE 5th International Symposium on Wireless Pervasive Computing 2010, no. 20. IEEE, 2010, pp. 295-300.

[15] J. Blumenthal, R. Grossmann, F. Golatowski, and D. Timmermann, "Weighted centroid localization in zigbee-based sensor networks," in 2007 IEEE international symposium on intelligent signal processing, no. 22. IEEE, 2007, pp. 1-6.

[16] Wikipedia, software defined radio. [Online]. Available: https://en. wikipedia.org/wiki/Software-defined_radio 